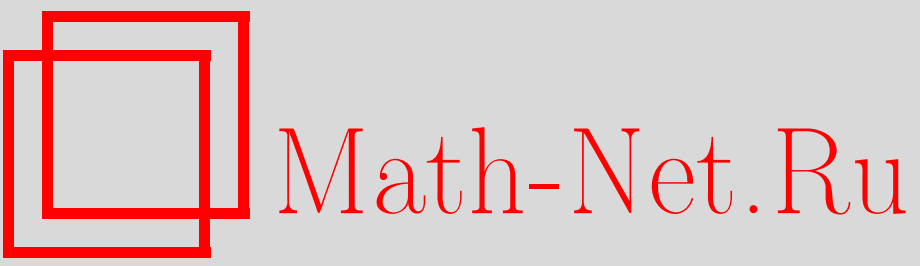

И. Б. Кожухов, О полугруппах с условиями на односторонние конгруэнции, УМН, 1998, том 53, выпуск 6, 251-252

DOI: https://doi.org/10.4213/rm99

Использование Общероссийского математического портала Math-Net.Ru подразумевает, что вы прочитали и согласны с пользовательским соглашением

http://www . mathnet.ru/rus/agreement

Параметры загрузки:

IP : 54.157 .27 .8

26 апреля 2023 г., 13:01:46 


\title{
О ПОЛУГРУППАХ С УСЛОВИЯМИ НА ОДНОСТОРОННИЕ КОНГРУЭНЦИИ
}

\author{
И. Б. Кожухов
}

Полугруппы, удовлетворяющие условию максимальности для правых (или левых) конгруэнций, изучались в работах [1]-[5]. В [5] было доказано, что коммутативная полугруппа удовлетворяет условию максимальности в том и толко том случае, если она конечно порождена. Группы с условием максимальности на правые (левые) конгруэнции - это в точности группы с условием максималшности для подгрупп. Строение этих групп может быть довольно сложным (см. примеры из гл. 9 в [6]), но все они, очевидно, являются конечно-порожденными. В [1] Хотцель поставил вопрос: является ли всякая полугруппа $S$ с условием максимальности для правых конгруәнций конечно порожденной? Им был дан положительный ответ в следующих случаях: а) когда $S$ слабо периодическая, т.е. $\forall a \in S \exists n\left(\left(S^{1} a^{n} S^{1}\right)^{2}=S^{1} a^{n} S^{1}\right)$, б) когда в $S$ всякий $J$-класс является классом некоторой конгруэнции (в частности, когда $S$ коммутативна). Примеры не конечно порожденных полугрупп с условием максимальности неизвестны, но если они существуют, их можно найти среди счетных полугрупп:

ПреДЛОЖЕнИЕ 1. Если существует полугруппа с условием максимальности для правых конгруәнций, не имеющая конечного множества образующих, то существует счетная полугруппа с тем же свойством.

Еще один результат о конечной порожденности дает следующая

Теорема 1. Если полугруппа удовлетворяет условию максимальности для правых и левьх конгруэнций, то она конечно порождена.

Известно, что в конечной полугруппе (более общо, в эпигруппе, т.е. в полугруппе, в которой некоторая степень каждого элемента принадлежит какой-нибудь подгруппе) отношения $\Gamma$ рина $D$ и $J$ совпадают (см. [7]), а значит, каждый $J$-класс является объединением попарно несравнимых $R$-классов. Для полугрупп с условием максимальности имеет место

ПрЕДЛОЖЕНИЕ 2. Каждый $J$-класс полугруппь с условием максимальности для правых конгруәнций является обвединением конечного числа попарно несравнимых $R$-классов.

Следующее утверждение показьвает, что в полугруппе с условием максимальности возможно вьполнение неравенства $D \neq J$.

ПРЕДЛОЖЕНИЕ 3. Пусть

$$
S=\left\langle a, s, t, u, 0 \mid a u=s^{2} a, a t u=a, t u=u t, a^{2}=a s=t a=u a=t s=s t=u s=s u=0\right\rangle .
$$

Тогда для любого поля $F$ полугрупповая алгебра $F S^{1}$ нётерова справа, а значит, $S$ удовлетворяет условию максимальности для правых конгруәнций. $B$ полугруппе $S$ $J_{a}=R_{a} \cup R_{s a}$, причем $R_{a}$ и $R_{s a}-$ различные $D$-классы.

Одно достаточное условие конечной порожденности дает следующее

ПредЛОЖЕНИЕ 4. Если $S$ - полугруппа с условием максимальности для правых конгруэнций и существует конечное подмножество $X$ такое, что подполугруппа $\langle X\rangle$ имеет непустое пересечение с каждым $L$-классом полугруппь $S$, то $S$ конечно порождена.

Полугруппа $S$ называется левой дуополугруппой, если в ней каждьй левьй идеал является двусторонним, т.е. $a S \subseteq S^{1} a$ для всех $a \in S$. Аналогично определяются правые дуополугруп$n b$. Положительный ответ на упоминавшийся ранее вопрос Хотцеля в случае дуополугрупп дает следуюшая 
Теорема 2. Если $S$ - правая или левая дуополугруппа, удовлетворяющая условию максимальности для правьх конгруэнций, то $S$ конечно порождена.

Пусть $\sigma$ - отношение эквивалентности на множестве $X$. Индексом отношения $\sigma$ называется мощность множества $X / \sigma$. (Если $\rho$ - правая на полугруппе $S$, то $S / \rho$ - правый $S$-полигон, но необязательно полугруппа.) Пусть $\Delta=\{(a, a) \mid a \in S\}$ - отношение равенства на полугруппе $S$. Для $a \in S$ положим

$$
\rho(a)=\left\{(x, y) \in S \times S \mid a^{i} x=a^{j} y \text { для некоторых } i, j\right\} .
$$

Условие максимальности для правых конгруэнций полугруппы $S$ будет обеспечено, если мы потребуем выполнения условия

$$
\text { всякая правая конгруэнция } \rho \neq \Delta \text { имеет конечньй индекс. }
$$

Класс полугрупп $S$, удовлетворяющих условию $(*)$, довольно узок: в [8] доказано, что всякая полугруппа с этим условием либо конечна, либо изоморфна подполугруппе аддитивной полугруппы $\mathbb{Z} \cup\{-\infty\}$. Более широкий класс составляют полугруппы $S$, удовлетворяющие следующему условию:

$$
\forall a \in S(\rho(a) \neq \Delta \Rightarrow|S / \rho(a)|<\infty) .
$$

Этому классу принадлежат: а) конечные полугруппы, б) нильполугруппы, в) полугруппы левых (правых) нулей, г) полурешетки с конечным нижним конусом (т.е. $|a S|<\infty$ для всех $a \in S$ ). Полугруппы с условием $(* *)$, конечно, могут не удовлетворять условию максимальности для правых конгруэнций. Однако, $(* *)$ является сильным ограничением на полугруппу, как показывает следующая

Теорема 3. Пусть $S$ - полугруппа с условием (**). Тогда:

(1) подполугруппа и гомоморфный образ полугруппь $S$ также удовлетворяют $(* *)$;

(2) всякая бесконечная подгруппа полугруппь $S$ является чиклической;

(3) $|e S|<\infty$ для любого идемпотента е, не являющегося левой единицей;

(4) всякий (0-)простой главный фактор полугруппь $S$ вполне (0-)прост;

(5) если $M^{0}(G, I, \Lambda, P)$ - вполне 0-простой главньй фактор полугруппь $S$, то либо $|G|,|\Lambda|<\infty$, либо $|I|,|\Lambda|<\infty u G \cong \mathbb{Z}$, либо $|G|=|I|=1 ;$ аналогичное утверждение верно для вполне простого главного фактора.

\section{СПИСОК ЛИТЕРАТУРЫ}

[1] Hotzel E. // Semigroup Forum. 1975-1976. V. 11. P. 337-362. [2] Hotzel E. // J. Algebra. 1979. V. 60. P. 352-370. [3] Kozhukhov I. B. // Semigroup Forum. 1980. V. 21. P. 337-350. [4] Zhang Liang, Gu Chang Kang. // World Sci. Words, Languages and Combinatorics, II (Kyoto, 1992). River Edge, NJ: World Scientific, 1994. P. 465-476. [5] Budach L. // Monatsberichte Deutschen Akad. Wiss. 1964. V. 6. P. 85-88. [6] Ольшанский А. Ю. Геометрия определяющих соотношений в группах. М.: Наука, 1989. [7] Клиффорд А., Престон Г. Алгебраическая теория полугрупп. Т. 1, 2. М.: Мир, 1972. [8] Кожухов И. Б. // Фундам. и прикл. матем. 1997. Т. 3. С. 869-878. 